\title{
A discovery strategy for novel cancer biomarkers
}

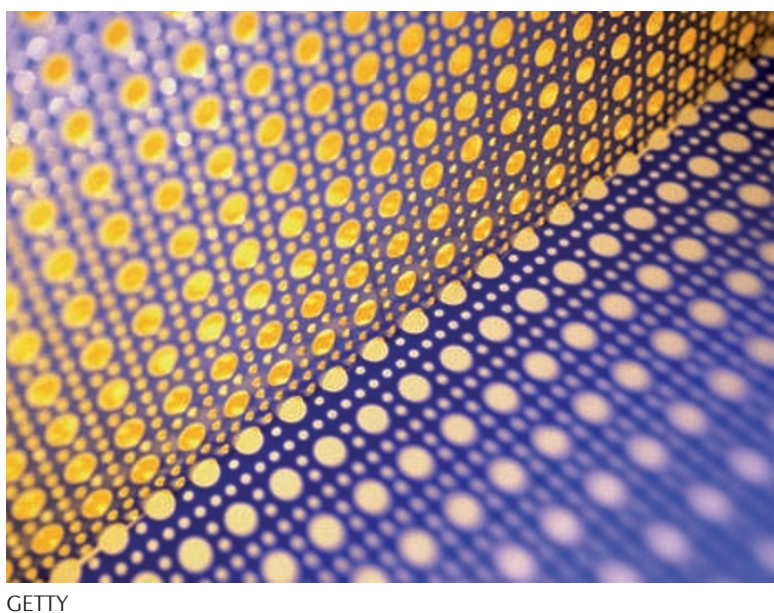

Patient responses to targeted therapy can be predicted by examining the modulation of oncogenic signalling pathways in response to specific drugs, which can also identify new pathway-specific biomarkers. The validity of this approach is illustrated in a new study that uses a proteomicsbased strategy to identify novel biomarkers that indicate a response of cancer cells to PI3K pathway inhibitors.

Andersen and colleagues used immunoaffinity precipitation followed by mass spectrometry to identify differential serine-threonine phosphorylation events in stable isotope labelling by amino acids in cell culture (SILAC)-labelled, PTENnull PC-3 prostatic cancer cells that were treated with inhibitors of PDK1, AKT or a dual inhibitor of PI3K and mTOR. This approach allowed the quantification of 375 phosphopeptides that were relevant to PI3K pathway signalling, of which 71 were drug inhibited and 11 were reduced by all 3 PI3K pathway inhibitors.

The authors identified 'PI3K pathway nodes', which were the phosphorylation targets that were the most reduced by drug treatment. For example, phosphorylation of the ribosomal protein RPS6 was the most strongly inhibited by all three drugs, and PRAS40 was the most reduced phosphopeptide by AKT and PI3KmTOR inhibition. To define common signalling modules, the authors analysed the phosphoproteins that were affected by all three inhibitors, and found that the modules most commonly inhibited were related to cytoskeletal functions, such as cell polarity and cytoskeletal organization. Additional modules commonly downregulated by the PDK1 and AKT inhibitors were related to vesicle transport and protein translation. These findings were consistent with the known inhibitory effects of these drugs on cancer cell motility, migration and invasion.

The authors identified phosphorylation sites that were uniquely downregulated by each PI3K inhibitor, several of which were novel. They suggested that these sites could be used as biomarkers for assessing responses to PI3K inhibitors and, as proof of principle, they evaluated the newly identified site phospho-Thr246-PRAS40 - which was reduced by the AKT inhibitor - as a biomarker. Andersen et al. generated an antibody against this epitope, and found that high levels of PRAS-Thr246 phosphorylation predicted sensitivity to an AKT inhibitor in lung and breast cancer cell lines. Moreover, the phosphoThr246-PRAS40 epitope is more stable than the phospho-Ser473AKT epitope that is commonly used in clinical diagnosis of tumours with AKT pathway activation, suggesting that this new biomarker might be more suitable for clinical evaluation.

Therefore, the signalling pathwaybased strategy described in this study allows the discovery of drug-specific biomarkers and has important clinical implications. The authors suggest that these biomarkers can be used to guide treatment decisions, and that globally analysing signalling pathways using a differential phosphoprofiling approach could be a way to assess the off-target effects of existing cancer drugs.

Meera Swami

ORIGINAL RESEARCH PAPER Andersen, J. N. et al. Pathway-based identification of biomarkers for targeted therapeutics: personalized oncology with PI3K pathway inhibitors. Sci. Trans. Med. 2 43a55 (2010)

FURTHER READING Kolch, W. \& Pitt, A. Functional proteomics to dissect tyrosine kinase signalling pathways in cancer. Nature Rev. Cancer 10, 618-629 (2010). 